\title{
Approaches and practices in developing teachers' expertise in mathematics instruction: an introduction
}

\author{
Yeping Li $\cdot$ Ruhama Even
}

Accepted: 12 October 2011 / Published online: 27 October 2011

(C) FIZ Karlsruhe 2011

\section{Background and purposes}

Teachers and their teaching have been recognized as a vital part of enhancing students' academic achievement (e.g., Sowder, 2007). While educational research has dramatically increased its emphasis on teachers and their practices over the past decades (e.g., Sikula, 1996; Townsend \& Bates, 2007), there is an ever-increasing need to improve teachers' expertise. Teachers' expertise can be improved in various ways, including practicing teachers' continuous knowledge and practice development in mathematics and pedagogy, teachers' training for undertaking and implementing changes in curriculum and instruction, and teachers' professional promotion. Although various approaches and practices (e.g., lesson study in Japan, teaching research group and apprenticeship practice in China, and video case-based learning in the US) have been generated and implemented to address different needs across educational systems, much remain to be learned about specific approaches and practices that have been developed and used effectively. Knowing and understanding effective approaches and practices used for developing teachers' expertise in mathematics instruction in different education systems have become especially important to those who care about

\footnotetext{
Y. Li (ه)

Department of Teaching, Learning and Culture, College of Education and Human Development, Texas A\&M University, College Station, TX 77843-4232, USA

e-mail: yepingli@tamu.edu

R. Even

Department of Science Teaching,

Weizmann Institute of Science, 76100 Rehovot, Israel

e-mail: ruhama.even@weizmann.ac.il
}

improving mathematics classroom instruction and teacher education.

In particular, proposing and editing this thematic issue were motivated with the following two reasons:

1. The thematic issue presents a fundamental belief about the unique value and importance of international collaboration and exchange of educational practices and research. Because both editors have extensive international experience, it has been clear to us that learning from others can not only allow us to learn what we do not know but also facilitate our reflection on what we already know. Efforts to improve educational practices can thus benefit from learning something new from others, as well as from revisiting and improving one's own practice. Recent large-scale international studies have led to the ever-increasing interest in learning from some high-achieving education systems, especially those in East Asia (e.g., Leung \& Li, 2010; Li \& Shimizu, 2009; Stigler \& Hiebert, 1999). Contributions from both the East and the West were thus invited and included. As readers should notice quickly from the structure of this thematic issue, articles about approaches and practices developed and used in the East are placed first and are followed by those contributed from the West.

2. The development and editing of this thematic issue is a sequel to a recent international collaborative publication on teachers' expertise in mathematics instruction (Li \& Kaiser, 2011). Although researchers have not come to a consensus on how to define and assess teachers' expertise (Lappan \& Li, 2002), the recent publication contributed to our understanding of different perspectives and values on teachers' expertise in different education systems (Li \& Kaiser, 2011). 
That book was the first step of taking a systematic examination of teacher expertise in mathematics instruction from an international perspective, and setting up a stage for the sharing and examination of different approaches and practices developed and used for improving teachers' expertise. As the development of teachers' expertise is part of teacher professional development in general, this thematic issue also builds on a recent international undertaking concerning the professional education and development of mathematics teachers (Even \& Ball, 2009). Similar to the intriguing differences noted in teacher professional development across different contexts (Even \& Ball, 2009), we expected to learn many different approaches and practices that are culturally valued for fostering excellent teachers and their expertise in different system and cultural contexts.

This issue, as a thematic collection of studies, consists of 18 articles that present and discuss different approaches and practices used to develop teachers' expertise in selected education systems in the East and the West (e.g., Australia, Chinese Mainland, Germany, Israel, Portugal, Singapore, South Korea, The Netherlands, and the United States) followed by a commentary article. This large number of papers reflects the ever-increasing interest and effort in improving teachers' expertise in different education systems. It presents an important contribution to the international community of mathematics education and teacher education, with contributions made by 40 scholars from 14 education systems. As editors of this thematic issue, we find ourselves continuously learning something new from our contributors. Such learning led us to believe that the articles published in this thematic issue are valuable sources of information for potential readers to learn and to further educational research and practices in developing teachers' expertise.

\section{Overview of the thematic issue}

As specified above, this thematic issue includes contributions from scholars about teacher expertise development in many education systems. Consistent with the structuring approach used in the recent book on expertise in mathematics instruction (Li \& Kaiser, 2011), we organize these articles as two clusters in terms of the two broad cultural regions: the East and the West. As a result, the thematic issue includes six articles (cluster 1) about approaches and practices in developing teachers' expertise in the East and 12 articles (cluster 2) about approaches and practices in developing teachers' expertise in the West. Within each cluster, the articles are organized in the order they were received. We hope that this structuring may facilitate readers to cross-examine possible similarities and differences within and across these two broad cultural regions, and that readers may be inspired to develop further research in the future.

The richness of these contributions is evident not only in terms of the number of educational systems that are represented but also in terms of the diverse perspectives and approaches that have been addressed in this thematic issue. Each article tends to focus on a certain aspect of teacher expertise and its development valued in a specific education system and selected by different researchers. No single article can simply be taken as to represent the approaches and practices used in one educational system. We expect that readers can gain much information about specific focuses and approaches in teacher expertise development from reading each article. At the same time, we suggest the following questions as a guideline to facilitate readers' discussion and cross-examination of the articles in this thematic issue:

1. What is the nature or aspect of teacher expertise that is being focused in this study? Is this nature/aspect of teacher expertise culturally specific or common to many other education systems?

2. What approach or practice for developing teacher expertise is being identified and examined in this study? Is this approach or practice culturally specific or adaptable to other education systems?

3. What theoretical perspective and/or research methods are used to conceptualize and document the effectiveness of specific approach or practice in this study?

In the following two sub-sections, we will provide a general overview of the articles that are placed in the two clusters.

2.1 Cluster 1: selected approaches and practices in developing teachers' expertise in mathematics instruction in the East

Six articles published in this thematic issue focus on approaches and practices developed and used to improve teachers' expertise in three education systems in the East: mainland China, Singapore, and South Korea. Although different contributors made their own choices to identify and examine specific approaches and practices, it is important to note that four articles are about approaches already in existence and two articles about researcherdeveloped programs. In particular, the three articles that focus on practices in Mainland China are all about different approaches and practices in existence. Li, Tang, and Gong (this issue) examined master teacher work stations for improving other experienced teachers' expertise that are now commonly in practice in Mainland China. Li and Qi 
(this issue) introduced and investigated the rapidly growing program of online study collaboration and its effects on teachers' expertise development in Mainland China. Huang and his colleagues (this issue) focused on exemplary lesson development and its effects on participating teachers' expertise growth in Mainland China. In addition to these three articles, Pang (this issue) focused on the case-based pedagogy used for developing prospective teachers' expertise in South Korea. In contrast, two articles contributed by scholars from Singapore are focused on their own programs designed and used for teachers' professional development. Leong and his colleagues (this issue) presented and discussed their project to build teachers' capacity to enact a problem-solving curriculum in a schoolbased design, whereas Kaur (this issue) presented a hybrid model of a school-based project for enhancing the pedagogy of mathematics teachers.

It is also important to point out that the three approaches/programs selected in Singapore and South Korea bear strong influences from the West. In contrast, the three approaches and practices identified in China clearly contain originality embedded in the Chinese culture of teaching and teacher professional development.

\subsection{Cluster 2: selected approaches and practices}

in developing teachers' expertise in mathematics instruction in the West

Scholars from ten education systems in the West contributed 12 articles about their approaches to and practices in teacher professional development. When we compare these articles with the articles about practices in the East, we can quickly discern one important difference. That is, almost all of the Western articles focus on approaches and practices that are researcher-designed programs/projects. For example, Clarke, Clarke, and Roche (this issue) outlined the benefits of task-based, one-to-one assessment interviews for building teachers' expertise in understanding, assessing and developing children's mathematical thinking. Their argument primarily built upon their own research and professional development project with teachers in Australia. Boston and Smith (this issue) presented a task-centric approach for teacher professional development in the United States. It is an approach that is developed from their on-going research and aimed at improving and sustaining teachers' ability to select and implement cognitively challenging mathematical tasks. Smit and van Eerde (this issue) presented and examined a teacher's learning in dual design research, to scaffold students' language development for mathematical learning in a multilingual mathematics classroom in The Netherlands. Their research led them to argue that dual design research is a fruitful way to promote and trace teacher expertise development. One exception is the article contributed by Even (this issue) that investigated in-service teachers' views about advanced mathematics studies after taking several advanced mathematics courses in a master's program. Overall, the apparent tendency to examine researcher-designed programs/projects suggests that scholars in the West are active researchers who always seek and develop ways to facilitate teachers' (expertise) development.

At the same time, the lack of non-researcher-designed approaches and practices identified and focused in this cluster can lead to the question about the ownership of these approaches and practices. It is often unclear how well these researcher-designed programs/projects are perceived and accepted by teachers as recipients. Possible differences in identified approaches and practices among the articles across these two clusters suggest possible cultural differences in developing and using approaches and practices for teacher (expertise) development. Further research and international collaborations are needed to examine and understand cultural support to teacher expertise development in the future.

\section{Significance and limitations}

This thematic issue aims to examine and share different approaches and practices developed and used in advancing teachers' expertise in mathematics instruction in selected education systems. With the inclusion of education systems from both the East and the West, this thematic issue provides mathematics educators a glimpse of various approaches and possible cultural resources utilized for developing teachers' expertise in mathematics instruction across selected education systems. It also provides educators an opportunity to reflect on possible approaches and practices developed and utilized in their own education systems. We hope that this thematic issue can serve as a starting point for further study in identifying and examining effective approaches and practices of developing teachers' expertise in mathematics instruction within and across different education systems.

At the same time, this thematic issue has its own limitations. One limitation is the unbalanced selection of education systems in the East and the West. It must be pointed out that these education systems were not selected for the purpose of comparing the East and the West, but because they represent a variety of different systems in the East and the West. We believe that, even with this limitation, the thematic issue identifies and examines specific approaches and cultural supports that are utilized to develop teachers' expertise in selected education systems in the East and the West. Another limitation is the inconsistence in selecting and focusing on different teacher populations 
(e.g., in-service teachers vs. pre-service teachers) across different studies. This limitation surely suggests readers not to make simple comparisons across different studies, but we also believe that the thematic issue provides rich and diverse information for readers to develop possible research in different directions.

Acknowledgments We want to thank all the contributors who worked diligently to meet the preparation and publication timelines. Special thanks go to all reviewers for reading manuscripts and providing valuable comments for improvements. Working with many colleagues from different education systems has been a very rewarding experience and underscores the international flavour of ZDM.

\section{References}

Even, R., \& Ball, D. L. (Eds.). (2009). The professional education and development of teachers of mathematics-the 15th ICMI Study. New York: Springer.
Lappan, G., \& Li, Y. (2002). Reflections and recommendations. International Journal of Educational Research, 37, 227-232.

Leung, F. K. S., \& Li, Y. (Eds.). (2010). Reforms and issues in school mathematics in East Asia-sharing and understanding mathematics education policies and practices. Rotterdam: Sense Publishers.

Li, Y., \& Kaiser, G. (Eds.). (2011). Expertise in mathematics instruction: An international perspective. New York: Springer.

Li, Y., \& Shimizu, Y. (Eds.). (2009). Exemplary mathematics instruction and its development in East Asia. ZDM-The International Journal on Mathematics Education, 41, 257-395.

Sikula, J. (Ed.). (1996). Handbook of research on teacher education (2nd ed.). New York: Macmillan.

Sowder, J. T. (2007). The mathematical education and development of teachers. In F. K. Lester Jr (Ed.), Second handbook of research on mathematics teaching and learning (pp. 157-223). Charlotte, NC: Information Age Publishing.

Stigler, J. W., \& Hiebert, J. (1999). The teaching gap: Best ideas from the world's teachers for improving education in the classroom. New York: Free Press.

Townsend, T., \& Bates, R. (Eds.). (2007). Handbook of teacher education: Globalization, standards and professionalism in times of changes. New York: Springer. 